\title{
Are young radio sources in equipartition?
}

\author{
M. Orienti ${ }^{1,2,3}$ and D. Dallacasa ${ }^{2,3}$ \\ 1 Instituto de Astrofisica de Canarias, 38200 La Laguna, Tenerife, Spain \\ 2 Dipartimento di Astronomia, Università di Bologna, via Ranzani 1, 40127 Bologna, Italy \\ 3 Istituto di Radioastronomia - INAF, via Gobetti 101, 40129 Bologna, Italy \\ e-mail: orienti@ira.inaf.it
}

Received 11 April 2008 / Accepted 21 May 2008

\begin{abstract}
Aims. The knowledge of physical conditions in young radio sources is important for defining the framework of models describing radio source evolution. We investigate whether young radio sources are in equipartition (i.e. minimum energy) conditions by comparing the equipartition magnetic fields of 5 High Frequency Peakers (HFP) with values directly inferred from the spectral peak assumed to be produced by synchrotron self absorption.

Methods. Multi-frequency VLBA observations of 5 HFPs were carried out in both the optically thick and thin part of the spectrum to determine the spectral shape and angular size of the components for which individual radio spectra were obtained.

Results. We find that the magnetic fields measured using observations agree well with those obtained by assuming equipartition, which implies that these sources are in minimum energy condition and the turnover in their spectra is due probably to SSA. In two source components, we found that the peak of the spectrum is caused by absorption of a thermal plasma instead of being due to SSA. The magnetic fields found in the various components range from 10 to $100 \mathrm{mG}$. In the presence of such high magnetic fields, electron populations with rather low $\gamma$ emit in the GHz-regime. In one source, we detect low-surface brightness extended emission at low frequency located $\sim 30$ mas $(\sim 50 \mathrm{pc})$ from the main source. This feature may be related to either an earlier episode of radio activity or a discontinuous start of the radio activity (sputtering). By comparing our data with previous VLBA observations, we estimate the hotspot advance speed to be in the range $0.1-0.7 c$ and kinematic ages of a few hundred years.
\end{abstract}

Key words. galaxies: active - radio continuum: general - magnetic fields - radiation mechanisms: non-thermal

\section{Introduction}

In a scenario where radio sources grow in a self-similar way, the evolution of each radio object originated by an Active Galactic Nucleus (AGN) depends on its linear size. It is established that the population of intrinsically compact radio sources represents an early stage of radio source evolution. In such a context, the empirical anti-correlation found between the linear size and the turnover frequency (O'Dea \& Baum 1997) implies that the youngest sources - with ages of about $\sim 100-1000$ years - must be sought among those whose turnover frequency occurs at a few GHz, which are termed "High Frequency Peakers" (HFPs, Dallacasa et al. 2000; Dallacasa 2003).

Several evolution models (e.g. Fanti et al. 1995; Readhead et al. 1996; Snellen et al. 2000) were proposed to describe how the physical parameters (i.e. luminosity, linear size and velocity) evolve as the radio emission grows within the host galaxy. Such evolution models are based on the assumption of minimum energy content, corresponding to a near equipartition of energy between the radiating particles and the magnetic field (Pacholczyk 1970) usually referred to as "equipartition". However, there is no a priori reason for believing that magnetic fields in radio sources are in equipartition. It is thus important to test the reliability of the equipartition at various stages of the radio source evolution; the study of newly born radio sources provides useful information about the physical parameters at the beginning of source growth. If the spectral peak is due to pure synchrotron selfabsorption (SSA) as claimed by Snellen et al. (2000), we have an independent way of estimating the magnetic field by measuring the frequency at which the radio emission changes from being opaque to transparent. In this case, the magnetic field $H$ can be measured directly from the spectral peak parameters, namely the peak frequency $v_{\mathrm{p}}$, peak flux density $S_{\mathrm{p}}$, and the source component angular sizes $\theta_{\text {maj }}$ and $\theta_{\text {min }}$ (Kellermann \& Pauliny-Toth 1981):

$H \sim f(\alpha)^{-5} \theta_{\mathrm{maj}}^{2} \theta_{\min }^{2} \nu_{\mathrm{p}}^{5} S_{\mathrm{p}}^{-2}(1+z)^{-1}$.

The function $f(\alpha)$ only weakly depends on $\alpha$, and for $\alpha=0.5$, $f(\alpha) \sim 8$ (Kellermann \& Pauliny-Toth 1981) ${ }^{1}$. The main difficulty in applying this method has been the uncertainty in determining source component parameters at the turnover frequency. Since the magnetic field depends strongly on these observable quantities, the estimates of the field strength derived so far are not very accurate. In particular, the uncertainties in the component size, a parameter that may be difficult to measure, contribute to the limited accuracy of the magnetic field estimates. Furthermore, magnetic fields should not be measured for the entire source but for its individual sub-structures, which are more likely to represent "homogeneous" components such as those studied in analytic models.

Scott \& Readhead (1977) and Readhead (1994) computed the magnetic field for sources of low-frequency spectral turnovers close in value to the observing frequency and found

\footnotetext{
1 Throughout this paper, the radio spectrum is described by the power law $S \propto v^{-\alpha}$.
} 
Table 1. Observational parameters of the HFP source components.

\begin{tabular}{|c|c|c|c|c|c|c|c|c|c|c|c|c|c|c|}
\hline $\begin{array}{c}\text { Source } \\
(1) \\
\end{array}$ & $\begin{array}{c}\text { Comp. } \\
\text { (2) }\end{array}$ & $\begin{array}{l}\text { Opt } \\
\text { (3) }\end{array}$ & $\begin{array}{c}z \\
(4) \\
\end{array}$ & $\begin{array}{c}S_{1.7} \\
\mathrm{mJy} \\
(5)\end{array}$ & $\begin{array}{c}S_{2.27} \\
\text { mJy } \\
(6)\end{array}$ & $\begin{array}{l}S_{5.0} \\
\text { mJy } \\
(7)\end{array}$ & $\begin{array}{c}S_{8.4} \\
\mathrm{mJy} \\
(8) \\
\end{array}$ & $\begin{array}{l}S_{15} \\
\text { mJy } \\
(9)\end{array}$ & $\begin{array}{l}S_{22} \\
\mathrm{mJy} \\
(10)\end{array}$ & $\begin{array}{c}v_{p} \\
\mathrm{GHz} \\
(11)\end{array}$ & $\begin{array}{c}S_{p} \\
\text { mJy } \\
(12)\end{array}$ & $\begin{array}{l}\theta_{\text {maj }} \\
\text { mas } \\
(13)\end{array}$ & $\begin{array}{l}\theta_{\min } \\
\text { mas } \\
(14)\end{array}$ & $\begin{array}{c}\text { PA } \\
\circ \\
(15) \\
\end{array}$ \\
\hline \multirow[t]{3}{*}{$\mathrm{J} 0003+2129$} & $\mathrm{E}$ & G & 0.45 & 101 & & 214 & 168 & $126^{a}$ & & $5.0 \pm 0.6$ & 220 & 0.41 & 0.12 & 140 \\
\hline & $\mathrm{Ce}$ & & & & & & 23 & & & & & 2.35 & 1.15 & 68 \\
\hline & W & & & & & 15 & 7 & $5^{a}$ & & & & 0.78 & - & 170 \\
\hline \multirow[t]{2}{*}{$\mathrm{J} 0005+0524$} & E & Q & 1.887 & 173 & & 110 & 60 & $23^{a}$ & & & & 0.81 & 0.80 & 97 \\
\hline & W & & & & & 79 & 103 & $82^{a}$ & & & & 0.66 & - & - \\
\hline \multirow[t]{3}{*}{$\mathrm{J} 0428+3259$} & $\mathrm{Ce}$ & G & 0.479 & 183 & 278 & 453 & 478 & 250 & $168^{a}$ & $5.9 \pm 0.1$ & 500 & 0.62 & 0.17 & 107 \\
\hline & E & & & 12 & 21 & 52 & 40 & 27 & $12^{a}$ & $6.2 \pm 0.9$ & 60 & 0.70 & 0.21 & 118 \\
\hline & W & & & & & & & 72 & $40^{a}$ & & & 0.32 & 0.25 & 150 \\
\hline \multirow[t]{3}{*}{$\mathrm{J} 0650+6001$} & $\mathrm{~N}$ & Q & 0.455 & 637 & 866 & 745 & 658 & 495 & $327^{a}$ & $6.35 \pm 0.01$ & 770 & 0.37 & 0.24 & 25 \\
\hline & $\mathrm{S}$ & & & & & 337 & 253 & 122 & $78^{a}$ & $3.1 \pm 0.1$ & 460 & 0.56 & 0.31 & 28 \\
\hline & Ext & & & & & 26 & 5 & 4 & & & & 2.3 & 0.4 & 20 \\
\hline \multirow[t]{4}{*}{$\mathrm{J} 1511+0518$} & E & $\mathrm{G}$ & 0.084 & & 27 & 127 & 224 & 242 & $150^{a}$ & $11.23 \pm 0.46$ & 250 & 0.43 & 0.16 & 80 \\
\hline & $\mathrm{Ce}$ & & & & & & 20 & 32 & & & & 0.27 & 0.13 & 60 \\
\hline & $\mathrm{W}$ & & & 94 & 191 & 484 & 458 & 449 & $285^{a}$ & $7.1 \pm 0.1$ & 500 & 0.56 & 0.13 & 70 \\
\hline & Ext & & & 3 & & & & & & & & 18 & 12 & 170 \\
\hline
\end{tabular}

Note 1. Observational parameters of the HFP source components. Column 1: source name (J2000); Column 2: component; Column 3: optical identification: $\mathrm{G}=$ galaxy, $\mathrm{Q}=$ quasar; Column 4: redshift; Columns 5, 6, 7, 8, 9 and 10: VLBA flux density at 1.7, 2.27, 5.0, 8.4, 15.3 and $22.2 \mathrm{GHz}$; Columns 11, 12 and 13: the deconvolved major and minor axis and position angle of the major axis. $a$ : Flux density from Orienti et al. (2006).

that the magnetic fields inferred directly from the spectrum were within a factor of 16 of the equipartition values. However, there are no systematic studies of sources with spectra peaking at higher frequencies, i.e. objects younger than those in Scott \& Readhead (1977).

Spangler et al. (1983) studied the magnetic field in the GHz-peaked spectrum (GPS) sources DA 193 (J0555+3948) and B1848 +283 , which were both classified as HFP by Dallacasa et al. (2000). They found that B1848+283 was in equipartition, while DA 193 was not. On the other hand, Orienti \& Dallacasa (2008a) investigated the magnetic field in the very young GPS object $\mathrm{J} 1459+3337$ and did not find any evidence of departure from equipartition. It can be considered to be one of the youngest objects known so far.

However, it is possible that the turnover in the spectrum is caused by free-free absorption (FFA) in the external ionized medium, as found for a number of young radio sources (e.g. Kameno et al. 2000; Marr et al. 2001). In this case, the magnetic field inferred from the peak would be physically meaningless.

We determine the magnetic fields of the components of 5 High Frequency Peaker radio sources from the Bright HFP sample (Dallacasa et al. 2000). The turnover frequency has a value of a few $\mathrm{GHz}$, corresponding to frequencies sampled well by VLBA observations; this implies that the magnetic field strength of these objects can be readily determined from spectral parameters and we can infer whether such objects are in equipartition.

Throughout this paper, we assume the following cosmology: $H_{0}=71 \mathrm{~km} \mathrm{~s}^{-1} \mathrm{Mpc}^{-1}, \Omega_{\mathrm{M}}=0.27$ and $\Omega_{\Lambda}=0.73$, in a flat Universe.

\section{Target sources}

The "bright" HFP sample (Dallacasa et al. 2000) consists of 55 candidate young radio sources selected on the basis of the their convex and high-frequency peaking radio spectrum. However, not all the objects are genuinely young radio sources but there is a significant fraction of contaminant blazar objects that match the selection criteria during a particular phase of their characteristic variability (e.g. Dallacasa et al. 2000).

Several studies were completed to determine the real nature of each object. For the majority of their lifetime, genuinely young radio sources and blazars have different properties. Young radio sources usually do not show significant spectral variability, and their radio emission - not exceeding the pc-scale and with a Double/Triple morphology dominated by lobes and/or hot-spots - is generally unpolarized. On the other hand, blazars are variable objects, and their emission, with a core-jet structure, is strongly polarized.

For this reason, we selected five of the HFPs that turned out to be genuine young radio sources on the basis of the results provided by investigations of spectral variability (Orienti et al. 2007; Tinti et al. 2005), morphology (Orienti et al. 2006), and polarization (Orienti \& Dallacasa 2008b).

The targets have a convex radio spectrum peaking at frequencies between $\sim 4$ and $15 \mathrm{GHz}$, and their pc-scale radio emission is resolved into 2-3 well-separated individual sub-components, each with a radio spectrum peaking at frequencies around a few $\mathrm{GHz}$ or higher. Therefore, these sources are ideal targets to investigate the physical conditions in young radio objects, as well as the intrinsic magnetic field of each sub-component.

The observed objects are listed in Table 1. Together with $\mathrm{J} 1459+3337$, they make up a small sample of 6 objects suitable for testing whether equipartition magnetic field may apply even in the earliest phase of the life of a radio source. The parameters for $\mathrm{J} 1459+3337$, listed in Table 2, are from Orienti \& Dallacasa (2008a).

\section{VLBA observations and data reduction}

The target sources were observed with the VLBA at 1.7 ( $L$ band), 5.0 ( $C$ band), and 15.3 ( $U$ band) in full polarization mode with a recording band width of $16 \mathrm{MHz}$ at $128 \mathrm{Mbps}$, while at 2.27 ( $S$ band) and $8.4 \mathrm{GHz}$ ( $X$ band) the dichroic receiver allowed a single polarization. Observations were carried out between 

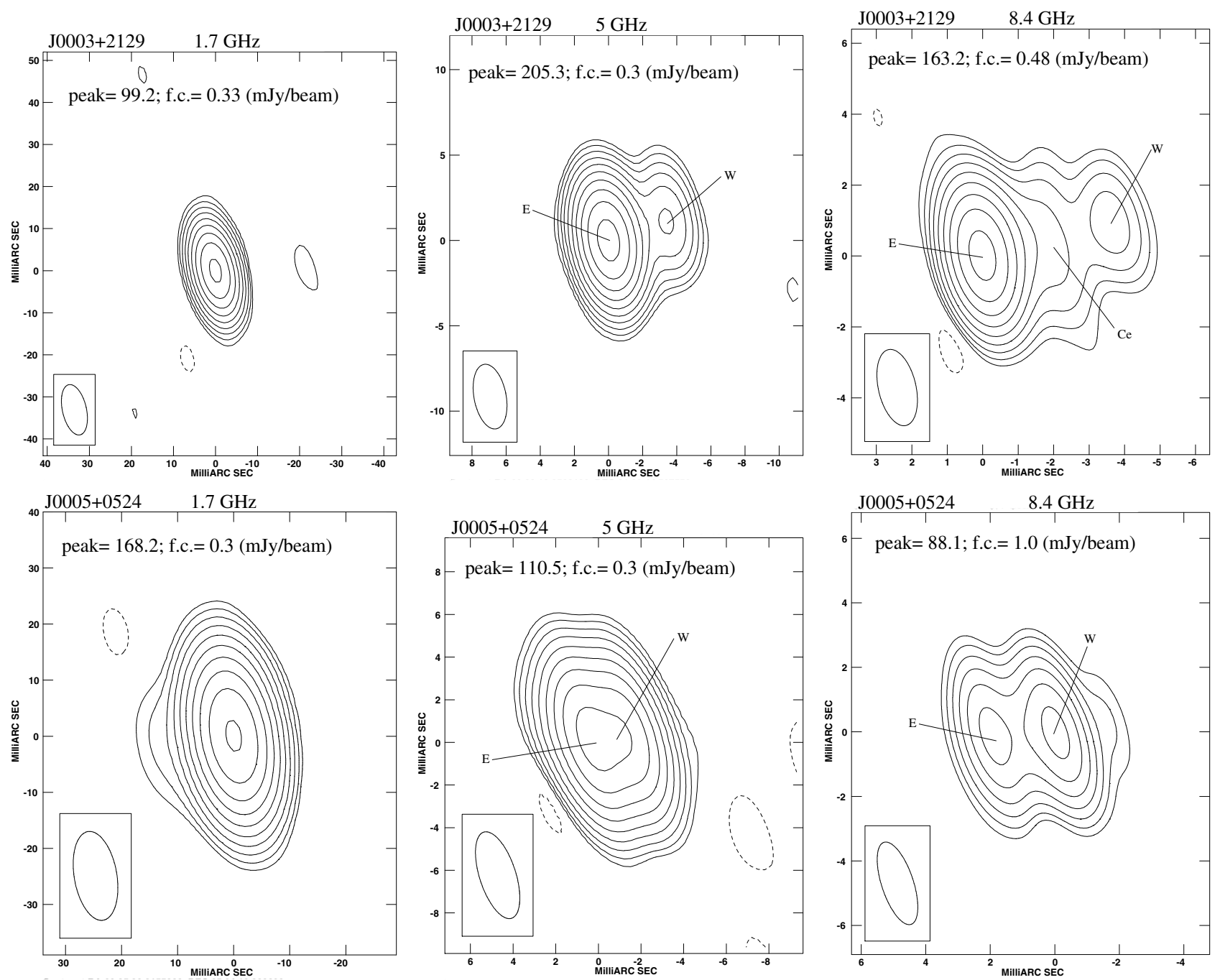

Fig. 1. VLBA images of the sources J0003+2129 (top) and J0005+0524 (bottom) in $L, C$ and $X$ bands. VLBA observations of these two sources were carried out on July 22 2006. For each image, we provide the following information on the plot itself: a) the source name and the observing frequency on the top left corner; b) the peak flux density in $\mathrm{mJy} / \mathrm{beam}$; c) the first contour intensity (f.c. in mJy/beam), which is usually 3 times the off-source rms noise level measured on the image; contour levels increase by a factor 2 ; d) the restoring beam, plotted on the bottom left corner.

July and August 2006 in five different runs, for a total time of $36 \mathrm{~h}$. The correlation was performed at the VLBA correlator in Socorro and the data reduction was carried out with the NRAO AIPS package. After the application of system temperatures and antenna gains, the amplitudes were checked using data on either 4C39.25 (J0927+3902) or DA 193 (J0555+3948), both sources having the flux density monitored at the VLA at 4.9 and $8.4 \mathrm{GHz}$, allowing the verification of the amplitude calibration. The error in the absolute flux density scale is generally within 3\%-10\%, being highest in value at the highest frequency.

In $C$ and $U$ bands, the instrumental polarization was removed by using the AIPS task PCAL; the absolute orientation of the electric vector of DA 193 and 4C 39.25 was compared with the VLA/VLBA polarization calibration database to derive the proper corrections. The values derived from the two sources were in good agreement $\left(<5^{\circ}\right)$.

For the sources J0003+2129 and J0005+0524, the system temperature measured in the $S$ band showed large variations and erratic values on short timescales, probably due to interference, and no reliable images could be obtained. At $15 \mathrm{GHz}$, the quality of data for these two sources, due to bad atmospheric transmittance, is low and significantly worse than the observations of Orienti et al. (2006).

The final images were obtained after a number of selfcalibration iterations. Amplitude self-calibration was applied only once at the end of the process, using particular care; the solution interval (30 min) was chosen to be longer than the scanlength to remove residual systematic errors and fine tune the flux-density scale, but not to force the individual data points to follow the model.

Full resolution images of the 5 HFP sources at the various frequencies are presented in Figs. 1, 2, 3, and 4. For each figure we provide the following information: the source name and the observing frequency on the top left corner; the peak flux density in $\mathrm{mJy} / \mathrm{beam}$; the first contour intensity (f.c. in mJy/beam), which is usually 3 times the off-source rms noise level measured on the image; contour levels increase of a factor 2 ; the restoring beam, plotted on the bottom left corner.

The final rms noises $(1 \sigma)$ are generally between 0.1 and $0.5 \mathrm{mJy} / \mathrm{beam}$. The dynamic range, defined as the ratio of the peak brightness to $1 \sigma$, is usually between 1000 and 3000 . 

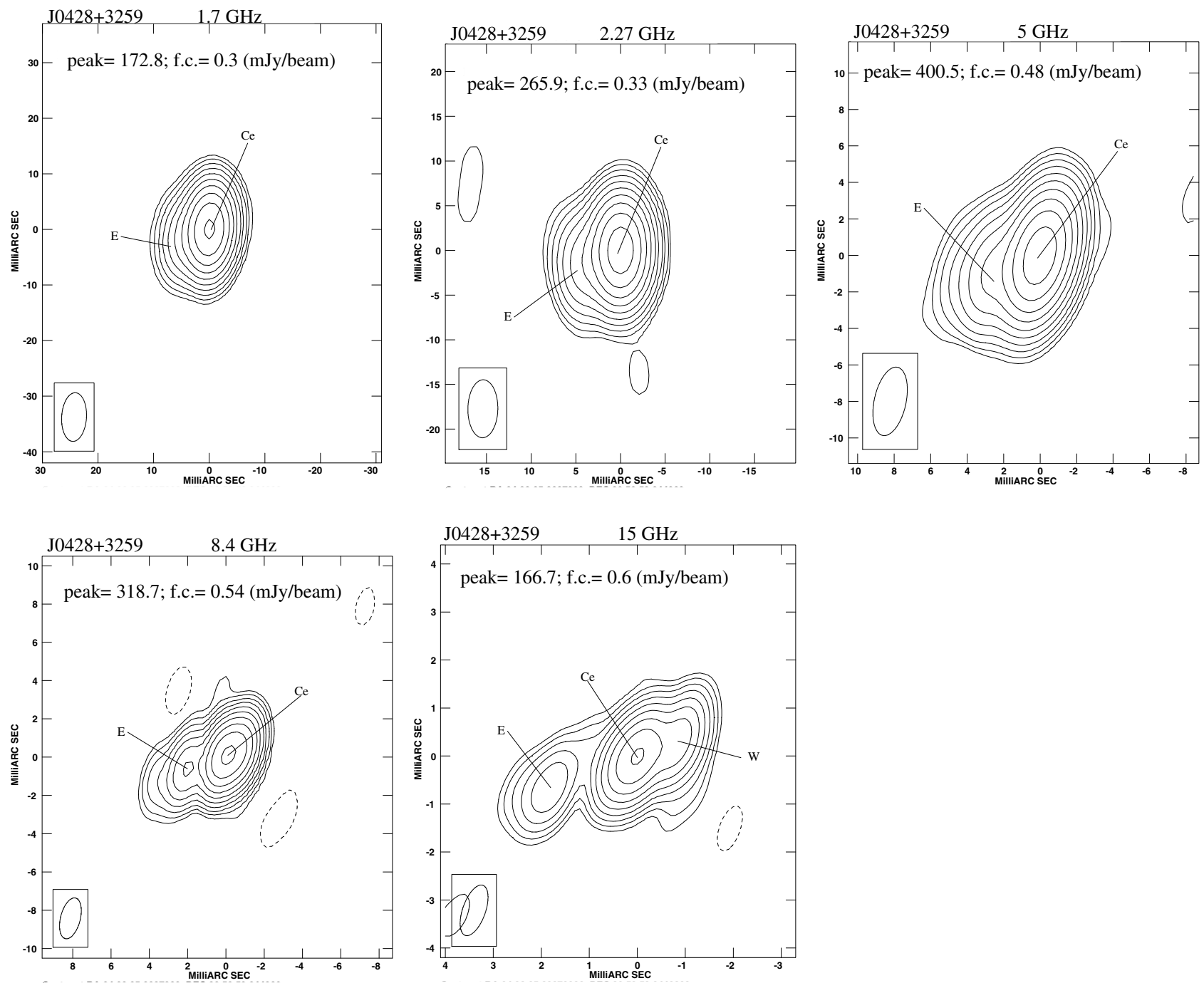

Fig. 2. VLBA images of the source J0428+3259 in $L, S, C, X$, and $U$ bands. This source was observed with the VLBA on August 172006 . For each image, we provide the following information on the plot itself: a) the source name and the observing frequency on the top left corner; $\mathbf{b})$ the peak flux density in $\mathrm{mJy} /$ beam; c) the first contour intensity (f.c. in mJy/beam), which is usually 3 times the off-source rms noise level measured on the image; contour levels increase by a factor 2 ; d) the restoring beam, plotted on the bottom left corner.

\section{Results}

Measurements of the source parameters with small uncertainties allow us to reliably derive the physical conditions in the target sources supposed to be in the earliest phase of the radio source evolution.

Source parameters, such as flux density and deconvolved angular sizes were measured by means of the task JMFIT, which performs a Gaussian fit on the image plane. The solutions were generally rather good, providing residuals consistent with the off-source noise. In case of extended components (e.g. $\mathrm{J} 1511+0518$ in $L$ band), the flux density was derived in the image plane by means of the tasks TVSTAT and IMSTAT.

Peak frequency and peak flux-density were determined by fitting the multi-frequency spectra of each source subcomponent with a pure analytical function (see e.g. Dallacasa et al. 2000). Where available, we considered the $22-\mathrm{GHz}$ data point from Orienti et al. (2006) to constrain more accurately the fit at high frequencies. Errors in peak frequency and peak flux density were calculated by error propagation theory: the former are reported in Table 1, while the latter account only for $\sim 1-3 \%$ of the peak flux density. In the case of the Eastern component of J0005+0524, the fit could not constrain the peak of the radio spectrum with sufficient accuracy and we do not derive the magnetic field using the parameters provided by the fit.

In Fig. 5, crosses indicate the VLBA spectra of subcomponents; plus signs show the total VLBA flux density obtained adding the spectra of each sub-component; diamonds represent the VLA overall flux density of Orienti et al. (2007). For a few source components, the availability of 3 or less data points did not allow us an accurate determination of the peak parameters, and they were not considered any further.

In Table 1, we report the observational parameters of each source component.

In the case of J0650+6001, the flux density at 1.7 and 2.27 was rescaled from Akujor et al. (1996). Errors associated with these extrapolations were conservatively to be $\sim 10 \%$.

No polarized emission was detected from the target sources at the mJy level in $C$ and $U$ bands, which is consistent with VLA measurements (Orienti \& Dallacasa 2008b). 

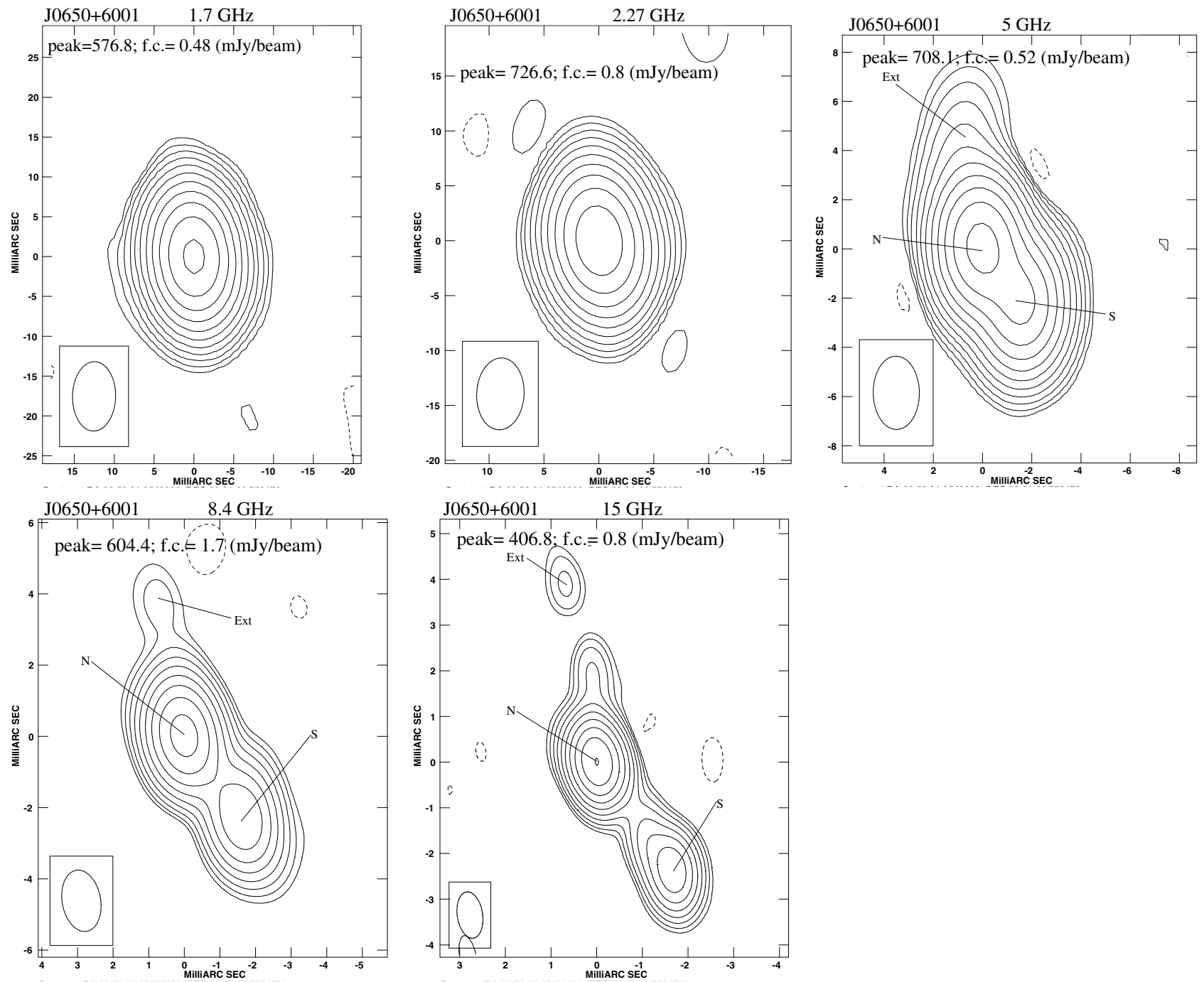

Fig. 3. VLBA images of the source J0650+6001 in $L, S, C, X$ and $U$ bands. VLBA observations were carried out on July 282006 . For each image, we provide the following information on the plot itself: a) the source name and the observing frequency on the top left corner; b) the peak flux density in $\mathrm{mJy} /$ beam; c) the first contour intensity (f.c. in mJy/beam), which is usually 3 times the off-source rms noise level measured on the image; contour levels increase by a factor 2 ; $\mathbf{d}$ ) the restoring beam, plotted on the bottom left corner.

\subsection{Source morphology}

The high-resolution achieved by these multi-frequency VLBA observations was adequate to resolve the HFP sources into a small number of sub-structures, each with its own radio spectrum and a rather accurate determination of source parameters (in particular the angular size) allowing us to constrain directly the magnetic field in a homogeneous component.

The observed sources were selected on the basis of their CSO-like morphology as found by Orienti et al. (2006) by means of observations in the optically-thin part of their radio spectrum. These new observations, carried out at several frequencies below and above the spectral peak, provide more information on the source sub-structures and their individual spectra.

The morphologies derived from these new observations are consistent with those found in previous observations, and a proper discussion of the source structures can be found in Orienti et al. (2006). We briefly summarize the information emerging from these new observations.

The galaxy J0003+2129, found to be Double by Orienti et al. (2006), displays a Triple structure in our new observations. The higher resolution achieved at $8.4 \mathrm{GHz}$ by the present data enabled us to identify a faint region located between the two main components at a distance of $\sim 1$ mas $(\sim 6 \mathrm{pc})$ from the brightest one.

The quasar J0005+0524 is characterized by a well defined Double structure. However, the two components have different characteristics: the spectrum of the Eastern component peaks at lower frequency than the Western one (Fig. 5), which is still selfabsorbed at $8.4 \mathrm{GHz}$ and becomes optically-thin at $\sim 15 \mathrm{GHz}$ (Orienti et al. 2006). At $1.4 \mathrm{GHz}$, there is a tentatively detection $(6 \sigma)$ of an extended emission accounting for $\sim 3 \mathrm{mJy}$.

The galaxy J0428+3259 shows a well defined Triple morphology, with a total linear size (LLS) of about $16 \mathrm{pc}$ and a position angle of $110^{\circ}$. All the source components have steep spectra and there is no indication of the source core.

The quasar J0650+6001 displays a Double structure, with an extended emission located at $\sim 4$ mas $(\sim 23 \mathrm{pc})$ and position angle of $\sim 10^{\circ}$ with respect to the Northern component. Such an extended feature can be resolved at 5.0, 8.4, and $15 \mathrm{GHz}$ only, in agreement with other works with similar resolution (see e.g. Stanghellini et al. 1999; Akujor et al. 1996).

The galaxy $\mathrm{J} 1511+0518$ is characterized by a well defined Triple structure. The availability of $8.4-\mathrm{GHz}$ observations at 

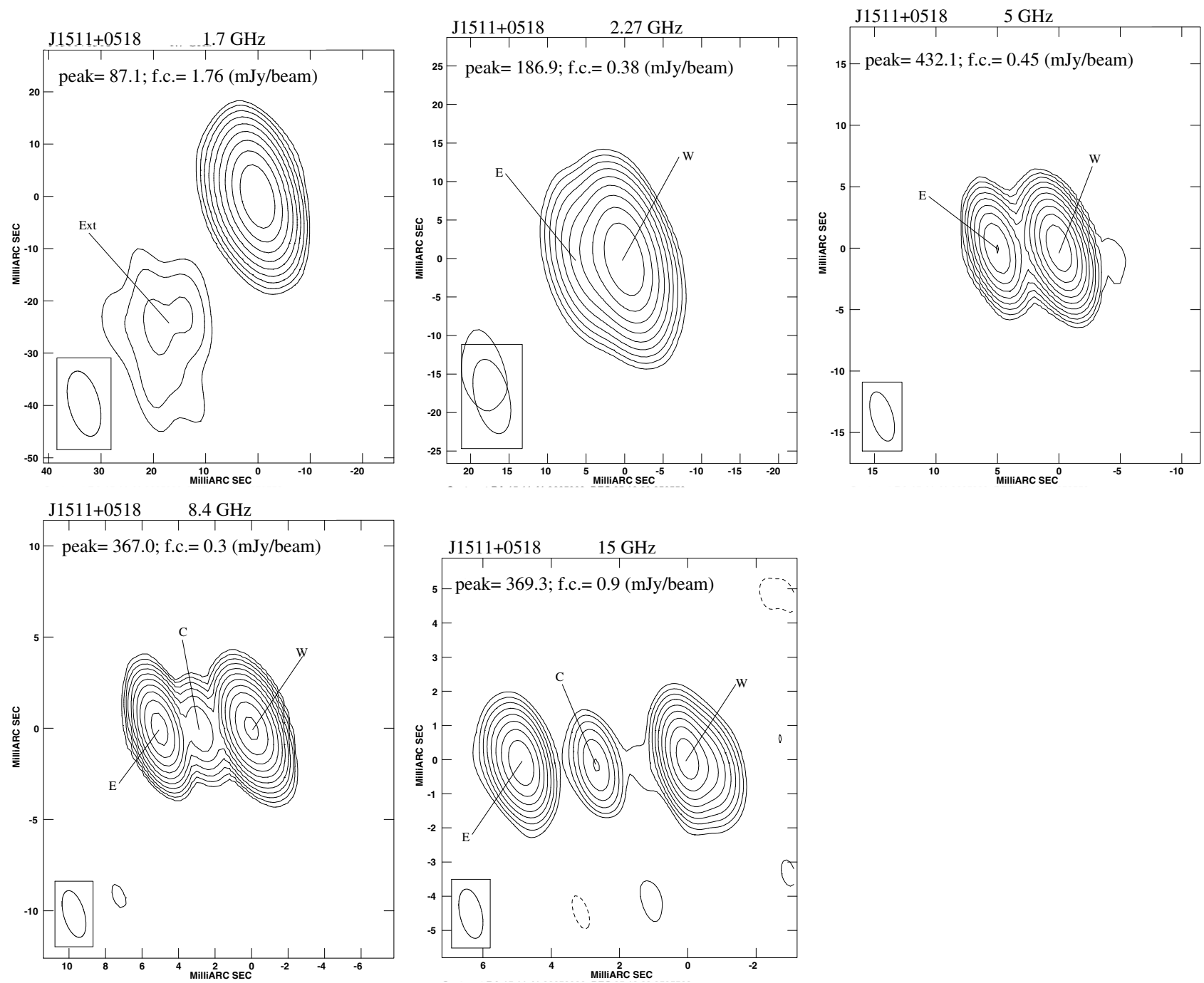

Fig. 4. VLBA images of the source J1511+0518 in $L, S, C, X$ and $U$ bands. VLBA observations were carried out on July 212006 . For each image, we provide the following information on the plot itself: a) the source name and the observing frequency on the top left corner; $\mathbf{b})$ the peak flux density in $\mathrm{mJy} / \mathrm{beam}$; c) the first contour intensity (f.c. in $\mathrm{mJy} /$ beam), which is usually 3 times the off-source rms noise level measured on the image; contour levels increase by a factor 2 ; $\mathbf{d}$ ) the restoring beam, plotted on the bottom left corner.

a higher spatial resolution than that achieved by Xiang et al. (2002) allows us to identify the central component as the source core, since it has an inverted spectral index $\alpha_{8.4}^{15} \sim-0.8$. At low frequencies, we find an additional extended emission, accounting for $\sim 3 \mathrm{mJy}$ at $1.7 \mathrm{GHz}$, located at $\sim 30$ mas $(\sim 50 \mathrm{pc})$ and oriented at a different position angle (p.a. $\sim 145^{\circ}$ ) with respect to the main source structure (p.a. $\sim 95^{\circ}$ ).

\subsection{Magnetic fields}

In the literature there are a few estimates of the magnetic field based on the determination of the spectral peak parameters. In general, difficulties in measuring the component angular size and peak frequency with good precision meant that it was not possible to derive accurate measurements of the magnetic field. The observing frequencies were not sufficiently close to the turnover frequency of the source components, leading to uncertainties in the determination of the magnetic field of a factor of ten (e.g. Rendong et al. 1991). Furthermore, complex source morphology and unsatisfactory $u v$ - and frequency coverages make this task extremely difficult. With its high spatial resolution, the VLBA is one of the best instrument to perform such an investigation, but nonetheless it has not been used for this purpose.

Since both the overall radio spectra of HFPs and the spectra of their single sub-components peak in the range of frequencies well sampled by the VLBA, we can constrain with good accuracy the turnover frequency, flux density, and angular size, and therefore achieve an accurate estimate of the magnetic field.

Following the approach by Readhead (1994) in Eq. (1) we consider component angular sizes that are 1.8 times larger than the full width at half maximum derived by the Gaussian fit. The uncertainty in the magnetic field measurement depends strongly on the accuracy of source parameters, in particular the angular size and peak frequency.

In general, we find that the magnetic field strengths derived from the observed spectral peak parameters are in the range of $\sim 10-100 \mathrm{mG}$ for the various components of the target sources discussed here, with typical uncertainties of a factor of 2 or even less. Two remarkable exceptions are J0428+3259 East and J1511+0518 East, for which we measured a magnetic field strength that was higher than a few Gauss. For these components, the radio peak appears to be inconsistent with pure synchrotron self-absorption and that a magnetic field derived by adopting this 

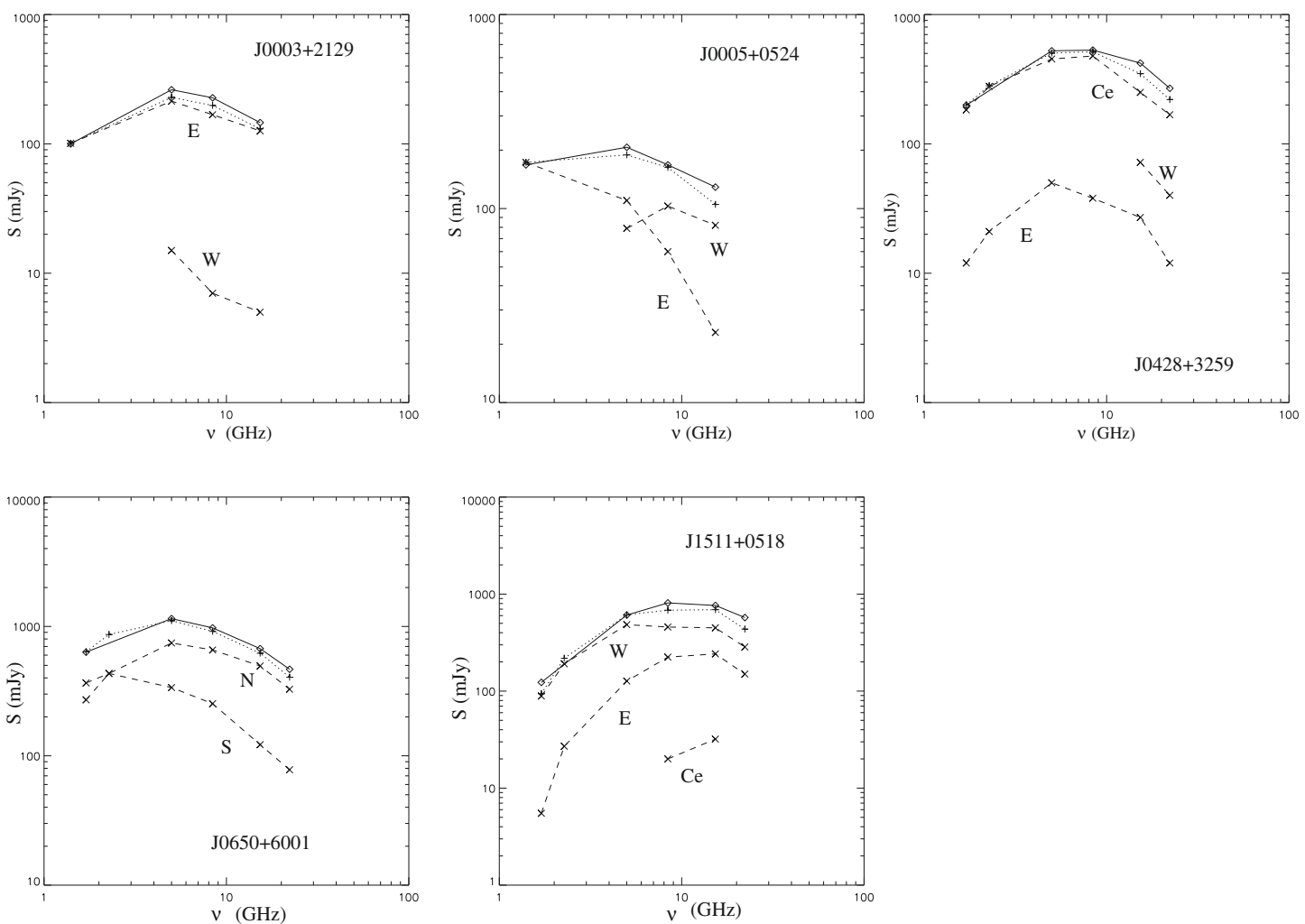

Fig. 5. Spectra of the 5 HFP sources and their sub-components. Crosses represent VLBA spectra of sub-components; plus signs show the total VLBA flux density obtained adding the spectra of each sub-component; diamonds represent the VLA overall flux density as from Orienti et al. (2007).

Table 2. Physical parameters of the source components.

\begin{tabular}{cccccc}
\hline \hline Source & $\mathrm{C}$ & $\begin{array}{c}H \\
\mathrm{mG}\end{array}$ & $\begin{array}{c}H_{\mathrm{eq}} \\
\mathrm{mG}\end{array}$ & $\begin{array}{c}u_{\min } \\
\mathrm{erg} / \mathrm{cm}^{3} \\
10^{-4}\end{array}$ & $\begin{array}{c}p_{\min } \\
\mathrm{dyne} / \mathrm{cm}^{2} \\
10^{-4}\end{array}$ \\
\hline $\mathrm{J} 0003+2129$ & $\mathrm{E}$ & 33 & 30 & 5.0 & 3.1 \\
$\mathrm{~J} 0005+0524$ & $\mathrm{E}$ & - & 18 & 0.75 & 0.46 \\
$\mathrm{~J} 0428+3259$ & $\mathrm{E}$ & $\gg 1000$ & 34 & 0.75 & 0.46 \\
& $\mathrm{Ce}$ & 59 & 65 & 3.9 & 2.4 \\
$\mathrm{~J} 0650+6001$ & $\mathrm{~N}$ & 29 & 77 & 6.0 & 4.0 \\
& $\mathrm{~S}$ & 10 & 54 & 1.5 & 1.0 \\
$\mathrm{~J} 1511+0518$ & $\mathrm{~W}$ & 104 & 95 & 8.3 & 5.2 \\
& $\mathrm{E}$ & $\gg 1000$ & 70 & 3.8 & 2.4 \\
$\mathrm{~J} 1459+3337$ & & 160 & 160 & 24 & 15 \\
\hline
\end{tabular}

assumption is unreliable (see Sect. 5.1 for a proper discussion). In the case of $\mathrm{J} 1511+0518$ East, this interpretation is supported by an optically-thick spectral index $<-2.5$, which is the lowest value achievable by SSA. In both components, FFA is detected against the faintest component. It is unclear whether this is due only to the presence of an additional absorber, which reduces the flux density.

We then derived the magnetic field by assuming that the source components were in equipartition. Equipartition magnetic fields were computed with standard formulae (Pacholczyk 1970), assuming an ellipsoidal geometry with a filling factor of unity (i.e. the source volume is fully and homogeneously filled with relativistic plasma). Furthermore, proton and electron energies were assumed to be equal. Equipartition values are accurately determined, and the field computed in this way was relatively insensitive to measured quantities that were poorly constrained by physical parameters. The equipartition magnetic fields obtained are reported in Table 2 with physical parameters such as the internal pressure and the energy density computed assuming a minimum energy condition (Pacholczyk 1970).

\subsection{Hot-spot separation velocity}

For the 5 HFPs presented here, the availability of VLBA observations carried out in 2002 enables us to estimate the hot-spot separation velocity.

The velocity determined in this way indicates the rate at which the working surfaces of the jets in the ISM are increasing their separation.

For the source $\mathrm{J} 0428+3259$, the uncertainties in the position of the components in the 2002 data are rather large and similar to the variation measured with respect to the 2006 data, preventing a significant estimate of the source growth based on these datasets only.

In the case of J1511+0518 where a central component is present, we computed the separation of the outer components with respect to the central one. The determination of the central component position in 2002 data was rather inaccurate ( 0.1 mas, i.e. the pixel size), and thus we prefer to derive only the hot-spot separation velocity.

We note that we measure separation velocities between two epochs only over a small time baseline, and the uncertainties in the estimated values are therefore high. When only data at two epochs are available, the errors cannot be estimated by regression analysis, and they are determined on the basis of the accuracy to which component positions can be measured (Polatidis \& Conway 2003). 
Table 3. Hot-spot separation velocity and the total linear size LLS.

\begin{tabular}{cccc}
\hline \hline Source & $v$ & LLS & age \\
& $c$ & pc & yr \\
\hline & & & \\
J0003+2129 & $0.15 \pm 0.10$ & 21 & 500 \\
J0005+0524 & $0.7 \pm 0.1$ & 15 & 140 \\
J0428+3259 & - & 16 & - \\
J0650+6005 & - & 17 & - \\
J1511+0518 & $0.10 \pm 0.01$ & 7 & 250 \\
\hline
\end{tabular}

For the sources J0003+2129 and J1511+0518, we found hotspot separation velocities of $0.15 c$ and $0.1 c$, respectively, similar to those obtained for the same class of objects, which are usually between $0.1 c$ and $0.4 c$. (Polatidis \& Conway 2003). In the case of J0005+0524, we measured a far higher velocity of $\sim 0.7 \pm 0.1 c$, while in $\mathrm{J} 0650+6001$ no hot-spot separation was detected, in agreement with previous works (e.g. Akujor et al. 1996). Among the young sources, this is the only object that does not show any evidence of proper motion so far.

We estimated the kinematic source age by assuming that the sources grow with a constant hot-spot separation velocity. Table 3 reports the hot-spot separation velocity, the total linear size (LLS), and the kinematic age of the observed sources.

\section{Discussion}

The direct measurement of the magnetic field in extragalactic radio sources is a difficult task to carry out. An indirect way to estimate the magnetic field is to assume that the radio source is in equipartition. This assumption was supported by equipartition brightness temperatures, which were found to agree with maximum brightness temperatures (Readhead 1994).

Alternatively, the magnetic field can be determined by comparing synchrotron and inverse Compton losses, but X-ray observations of small and young radio sources have not provided strong constraints so far, mainly due to the poorer spatial resolution of the X-ray telescopes with respect to that achieved by radio interferometry.

Another way to test whether radio sources are in equipartition is to compute their magnetic fields by means of observable quantities. In sources showing SSA, the value of the field strength can be derived directly from the synchrotron peak parameters.

In the following discussion, we investigate the physical conditions occurring in young radio sources by comparing the results obtained by using the peak parameters and those assuming equipartition conditions.

\subsection{Magnetic fields and the origin of the turnover}

For each source component, the magnetic fields that we estimated from the spectral peak determined from the data (i.e. frequency and flux density) range between 10 and $100 \mathrm{mG}$, in agreement with values found in case of equipartition, with the exception of J0428+3259 East and J1511+0518 East. Such a result suggests that young radio sources are in equipartition and the peak in their spectra is probably due to synchrotron selfabsorption. Only in J0428+3259 East and J1511+0518 East, a field higher than a few Gauss clearly exceeds the equipartition value. In the presence of such a high magnetic field, the
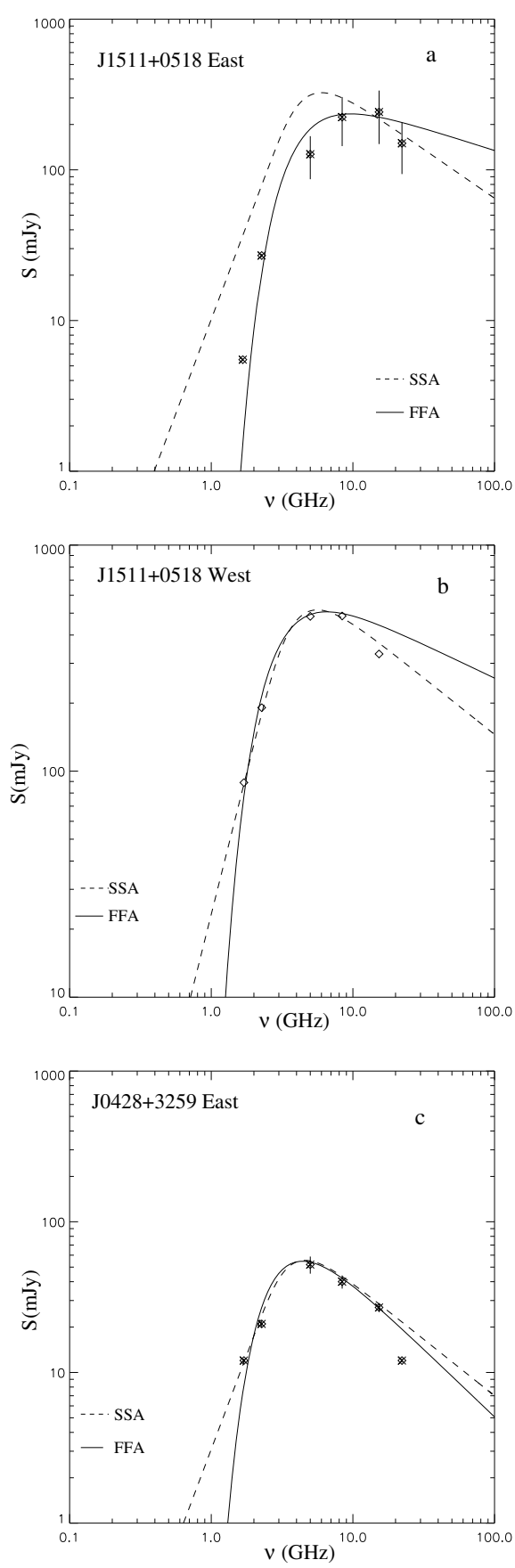

Fig. 6. FFA and SSA fit to the East (top) and West (middle) components of J1511+0518 and to the East component of J0428+3259 (bottom).

radiative lifetime $t_{\mathrm{rad}}$ of the electron population should be extremely short:

$t_{\mathrm{rad}} \sim 0.625 H^{-3 / 2} v_{\mathrm{br}}^{-1 / 2} \quad(\mathrm{yr})$

where $v_{\text {br }}$ is the break frequency in GHz and $H$ in Gauss. The radio spectrum of $\mathrm{J} 1511+0518$ East does not show any evidence of the spectral break in the frequency range spanned by the observations. If we assume a lower limit of $22 \mathrm{GHz}$ for the break frequency, we find that, in the presence of such high magnetic fields the electron population would have synchrotron ages $t_{\text {rad }} \sim 1 \mathrm{yr}$. However, the spectral coverage in the optically thin regime is rather sparse, preventing a proper detection of the break. 
A more realistic explanation is that the spectral peak is due to free-free absorption. To test the reliability of this hypothesis, we fit the spectrum with both FFA and SSA. From Fig. 6a, it is clear that the spectrum is described more accurately by a FFA model (solid line) than a SSA one (dashed line). This result, together with an optically-thick spectral index $<-2.5$, implies that the spectrum would be more accurately described by absorption by thermal plasma in front of the component, instead of simple synchrotron self-absorption alone. On the other hand, the spectrum of the the western component of J1511+0518 may be reproduced well by a pure SSA model without any additional contribution from FFA. The good agreement between the magnetic field obtained by the peak parameters and the equipartition value suggests that in this case the spectral peak is probably due to SSA. This result may indicate that the thermal plasma causing the absorption in the East component does not embed homogeneously the radio source, as in the presence of a diffuse and isotropic circumnuclear structure, but is probably due to a inhomogeneous ambient medium of different opacity along the various lines of sight, as also suggested by Kameno et al. (2000) in the case of the other HFP galaxy J1407+2827 (OQ 208).

The same approach was carried out to model the East component of J0428+3259 (Fig. 6c). The magnetic field inferred from the peak parameters was $H \sim 10 \mathrm{G}$, which is, again, substantially higher than for the components of the other target sources. Even for this component it is therefore likely that the spectral peak is due to absorption from an ionized ambient medium in addition to synchrotron self-absorption.

The discovery of free-free absorption in two of the five sources observed here may indicate a detection rate of such an effect that is higher in HFPs than in the larger GPS sources. The statistic is still limited and further observations are required, but this evidence is consistent with the idea that the smallest sources (i.e. HFPs) reside within the innermost region of the host galaxy, characterized by an extremely dense and inhomogeneous ambient medium of high electron density. Other detections of free-free absorption occurring in the HFP galaxies J0111+3906 (Marr et al. 2001) and J1407+2827 (alias OQ 208, Kameno et al. 2000) support this view.

In such a context, we cannot exclude that these components are in equipartition since we do not have another way to derive independently the magnetic field. Ideally, it may be measured by comparing synchrotron and inverse Compton losses, but Xray observations available cannot provide a comparable spatial resolution and the magnetic field derived would be averaged over the entire source volume and not over individual components.

However, to estimate the optical depth $\tau$ of the ionized medium we compute the peak frequency of these two components by assuming equipartition, since the other targets do not show any significant departure from minimum energy conditions. We find $v_{\mathrm{p}} \sim 1.9$ and $5.0 \mathrm{GHz}$ for J0428+3259 East and $\mathrm{J} 1511+0518$ East respectively, in the case of SSA only. Then we determine the optical depth $\tau$ by comparing the observed flux density $S_{\text {FFA }}$ with that obtained by extrapolating the spectral index of the optically-thin spectrum down to the peak $\left(S_{\mathrm{SSA}}\right)$ :

$\tau=-\ln \frac{S_{\mathrm{FFA}}}{S_{\mathrm{SSA}}}$

and the values derived (Table 4) are similar to those found in the components of the young GPS radio source B1946+708 (Peck et al. 1999).
Table 4. Free-free optical depth of J0428+3259 East and J1511+0518. The optical depth $\tau$ has been computed from Eq. (3).

\begin{tabular}{cccccc}
\hline \hline Source & $\alpha_{\text {thin }}$ & $\begin{array}{c}v \\
\mathrm{GHz}\end{array}$ & $\begin{array}{c}S_{\text {FFA }} \\
\text { mJy }\end{array}$ & $\begin{array}{c}S_{\text {SSA }} \\
\text { mJy }\end{array}$ & $\tau$ \\
\hline J0428+3259 E & 0.6 & 2.27 & 21 & 84 & 1.3 \\
& 0.6 & 5.0 & 50 & 55 & 0.09 \\
J1511+0518 E & 0.8 & 5.0 & 127 & 515 & 1.4 \\
& 0.8 & 8.4 & 224 & 340 & 0.4 \\
\hline
\end{tabular}

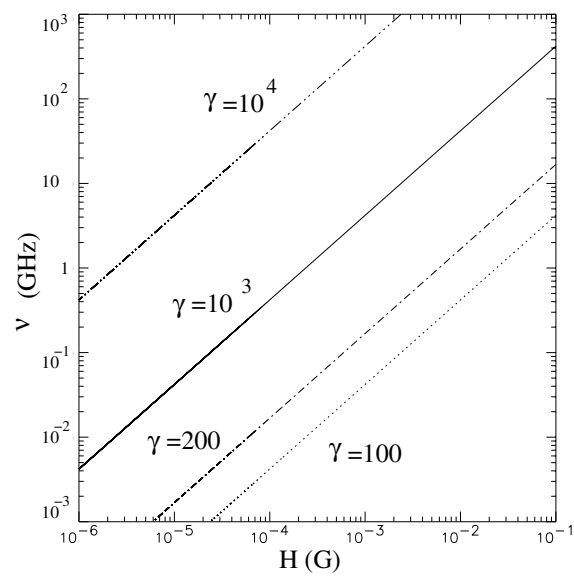

Fig. 7. Critical frequency emitted by electron populations with various $\gamma$ versus the magnetic field. $\gamma$ has been computed from Eq. (4).

\subsection{Magnetic field and the electron population}

As shown in the previous section, the magnetic fields obtained following equipartition and, when possible, confirmed by the value inferred from the peak parameters are in the range of $\sim 10 \mathrm{mG}$ up to $\sim 160 \mathrm{mG}$, if we also consider GPS J1459+3337 (Table 2). With such strong fields, electron populations with relatively small $\gamma$ can radiate at high frequencies. The critical frequency $v_{\mathrm{cr}}$ at which an electron with a certain $\gamma$ radiate is given by:

$v_{\mathrm{cr}} \sim 4.2 \times 10^{-3} \gamma^{2} H(\mathrm{GHz})$

(Pacholczyk 1970), where $H$ is in Gauss. From Eq. (4), it is clear that electrons with small $\gamma(\sim 200-500)$ can radiate at frequencies higher than a few $\mathrm{GHz}$ in the presence of $H$ in the range of $10-100 \mathrm{mG}$.

If we assume that the jet power output $P_{\mathrm{j}}$ is constant during the lifetime of the radio source and that the magnetic field is frozen into the plasma, we find that the magnetic field intensity decreases as the source grows:

$H=\left(\frac{P_{\mathrm{j}} t}{V}\right)^{1 / 2}$

where $t$ is the source age and $V$ is the volume of the lobe computed by assuming an ellipsoidal geometry and a filling factor of unity.

The decrement of the magnetic field as the source grows in size is in agreement with the observations: compact (LLS « $20 \mathrm{kpc}$ ) objects have magnetic fields of a few $\mathrm{mG}$ (e.g. Dallacasa et al. 2002; Fanti et al. 1995), while in large (LLS $\sim 1 \mathrm{Mpc}$ ) radio sources Murgia et al. (2001) found a magnetic field of a few $\mu \mathrm{G}$.

From Eq. (4), we see that the critical frequency at which an electron with a given $\gamma$ radiates, decreases with the magnetic field (Fig. 7). For example, in the presence of high magnetic 
fields ( $H \geq 10 \mathrm{mG}$ ), such as those found in compact objects, an electron with $\gamma \sim 1000$ emits at a frequency around $40 \mathrm{GHz}$, while in weaker fields $(H \sim 2 \mu \mathrm{G})$ the radiation is at a frequency about of $\sim 10 \mathrm{MHz}$, which is lower than the frequencies of the surveys carried out so far. As a consequence, during the evolution of a radio source the field intensity progressively decreases and the electrons radiating at a given frequency are those with higher and higher $\gamma$. For example,electrons with $\gamma \sim 350$ emit at $5 \mathrm{GHz}$ in the presence of $H=10 \mathrm{mG}$, while for $H=2 \mu \mathrm{G}$ only electrons with $\gamma \sim 24000$ are still visible at such a frequency. It is unclear whether all the small sources may become efficient accelerators to achieve $\gamma \sim 10^{3-4}$ as observed in extended radio sources. On the other hand, electrons with such energies, in the presence of a magnetic field $H \sim 0.1 \mathrm{G}$, radiate at $v_{\mathrm{cr}} \sim 2.5 \times 10^{14} \mathrm{~Hz}$, i.e. in the near-IR. Their contribution to the NIR emission is however limited, well below $1 \mathrm{mJy}$, as can be seen easily by extrapolating the optically thin spectra for all the sources presented here. At such a frequency and with such a high magnetic field, their radiative lifetime is extremely short and any synchrotron emission would be strongly contaminated by the thermal emission from the host galaxy. All this may provide further explanation for the open question concerning the high number counts of young radio sources with respect to the larger ones, if we assume that some of young radio sources will never be able to accelerate electrons to $\gamma>10^{3}$.

\subsection{Extended emission and discontinuous radio activity}

Another intriguing aspect of these young radio sources is the presence of low-surface brightness extended emission visible at low frequencies on the pc-scale. In the source J1511+0518, we detected low-surface brightness emission located about $50 \mathrm{pc}$ from the main source at a position angle of $110^{\circ}$ (Fig. 4). Extended emission on the pc-scale was discovered in the HFP galaxy J1407+2827 (Luo et al. 2007). Extended emission on the kpc-scale and beyond was already known in this class of objects (e.g. the HFP galaxy J0111+3906, Baum 1990), and interpreted in terms of the relic of a past radio activity which occurred about $10^{7}-10^{8}$ years ago. Extended features located at a pc-scale distance from the central object may be the relic of a far more recent previous activity that occurred about $10^{3}-10^{4}$ years ago.

Evidence of a "recent" earlier start may suggest that at the very beginning of the radio activity several cycles of subsequent short bursts (sputtering) occurred before the classical large Double radio sources started to develop. This was also suggested by Gugliucci et al. (2005), who estimated source ages of a few hundred years for the CSOs studied. The age distribution found peaks around 500 years, suggesting that either many CSOs die young or that they are a transient phenomenon and only a few become large Doubles (Gugliucci et al. 2005). Such ages agree with those derived from the hot-spot separation velocities measured in our sources. However, our values are affected by large uncertainties: a more robust estimate of the velocity separation and the kinematic age would need more observing epochs distributed over several years.

\section{Conclusions}

We have presented the results of multi-frequency VLBA observations of 5 young High Frequency Peakers. By assuming that the turnover of the radio spectrum is due to synchrotron selfabsorption, we derived the magnetic field of each source subcomponent by means of observable quantities, namely the peak frequency, peak flux-density, and the angular size. The magnetic fields calculated in this way are usually in good agreement with those obtained by assuming equipartition, with two exceptions. This suggests that in general young radio sources are in minimum energy conditions and their spectral turnover is caused by SSA. The values found for the magnetic fields are in the range of $10 \mathrm{mG}$ up to $\sim 160 \mathrm{mG}$, if we consider also the GPS source $\mathrm{J} 1459+3337$.

In two sub-components, we found evidence that the turnover of the spectrum is probably due to absorption by a thermal plasma located in front of the component, instead of SSA. The other components of these sources do not show any evidence of departure from SSA, suggesting that the absorber is an inhomogeneous ambient medium with different free-free opacities along the lines of sight to the two lobes.

In young radio sources, the presence of such high magnetic fields implies that even electron populations with small $\gamma$ (e.g. $\gamma \sim 200)$ radiate at the "high" frequencies sampled by the available surveys. This may provide a further explanation of the high number counts of young radio sources with respect to the larger ones.

In HFP J1511+0518 at $1.7 \mathrm{GHz}$, we unambiguously detected a low-surface brightness extended emission not aligned with the main source structure. Such a feature may be the relic of a previous epoch of radio activity that occurred not long ago $\left(<10^{4}\right.$ years $)$. This may be consistent with a scenario of a discontinuous start (sputtering) of the radio activity.

The analysis of the hot-spot separation velocity provided kinematic ages of a few hundreds of years, confirming that the targets can be considered young radio sources.

Acknowledgements. The VLBA is operated by the US National Radio Astronomy Observatory which is a facility of the National Science Foundation operated under a cooperative agreement by Associated University, Inc. This work has made use of the NASA/IPAC Extragalactic Database (NED), which is operated by the Jet Propulsion Laboratory, California Institute of Technology, under contract with the National Aeronautics and Space Administration.

\section{References}

Akujor, C. E., Porcas, R. W., \& Smoker, J. V. 1996, A\&A, 306, 391 Baum, S. A., O’Dea, C. P., de Bruyn, A. G., \& Murphy, D. W. 1990, ApJS, 74, 389

Dallacasa, D. 2003, PASA, 20, 79

Dallacasa, D., Stanghellini, C., Centonza, M., \& Fanti, R. 2000, A\&A, 363, 887 Dallacasa, D., Tinti, S., Fanti, C., et al. 2002, A\&A, 389, 115

Fanti, C., Fanti, R., Dallacasa, D., et al. 1995, A\&A, 302, 317

Gugliucci, N. E., Taylor, G. B., Peck, A. B., \& Giroletti, M. 2005, ApJ,622, 136 Kameno, S., Horiuchi, S., \& Shen, Z.-Q. 2000, PASJ, 52, 209

Kellermann, K. I., \& Pauliny-Toth, I. I. K. 1981, ARA\&A, 19, 373

Luo, W.-F., Yang, J., Cui, L., Liu, X., \& Shen, Z.-Q. 2007, ChJAA, 7, 611

Marr, J. M., Taylor, G. B., \& Crawford, F., III 2001, ApJ, 550, 160

Murgia, M., Parma, P., \& de Ruiter, H. R. 2001, A\&A, 380, 102

Orienti, M., \& Dallacasa D. 2008a, A\&A, 477, 807

Orienti, M., \& Dallacasa D. 2008b, A\&A, 479, 409

Orienti, M., Dallacasa, D., Tinti, S., \& Stanghellini, C. 2006, A\&A, 450, 959

Orienti, M., Dallacasa, D., \& Stanghellini, C. 2007, A\&A, 475, 813

O’Dea, C. P., \& Baum, S. A. 1997, AJ, 113, 148

Pacholczyk, A. G. 1970, Radio Astrophysics (San Francisco: Freeman \& Co.)

Peck, A. B., Taylor, G. B., \& Conway, J. E. 1999, ApJ, 521, 103

Polatidis, A. G., \& Conway, J. E. 2003, PASA, 20, 69

Readhead, A. C. S. 1994, ApJ, 426, 51

Readhead, A. C. S., Taylor, G. B., Xu, W., et al. 1996, ApJ, 460, 612

Rendong, N., Schilizzi, R. T., Fanti, C., \& Fanti, R. 1991, A\&A, 252, 513

Scott, M. A., \& Readhead, A. C. S. 1977, MNRAS, 180, 539

Snellen, I. A. G., Schilizzi, R. T., Miley, G. K. et al. 2000, MNRAS, 319, 445

Spangler, S. R., Mutel, \& R. L., Benson, J. M. 1983, ApJ, 271, 44

Stanghellini, C., O’Dea, C. P., \& Murphy, D. W. 1999, A\&A, 134, 309

Tinti, S., Dallacasa, D., de Zotti, G., Celotti, A., \& Stanghellini, C. 2005, A\&A, 432,31

Xiang, L., Stanghellini, C., Dallacasa, D., \& Haiyan, Z. 2002, A\&A, 385, 768 